\title{
Midlife Women's Responses to a Hospital Sleep Challenge: Aging and Menopause Effects on Sleep Architecture
}

\author{
JANE L. LUKACS, Ph.D., M.S.N., ${ }^{1}$ JULIE L. CHILIMIGRAS, M.P.H., ${ }^{1}$ \\ JASON R. CANNON, B.S., ${ }^{2}$ SHARON L. DORMIRE, Ph.D., M.S.N., ${ }^{4}$ \\ and NANCY E. REAME, Ph.D., M.S.N. ${ }^{1,3}$
}

\begin{abstract}
Objective: To distinguish aging from menopause effects on sleep architecture, we studied an episode of disturbed hospital sleep in asymptomatic midlife women during the follicular phase of an ovulatory cycle and three control groups differing by age or menopause status.

Methods: Fifty-one studies were conducted in four groups of volunteers: young cycling (YC, 20-30 years, $n=14$ ), older cycling (OC, 40-50 years, $n=15)$, ovariectomized receiving estrogen therapy (OVX, 40-50 years, $n=12)$, and spontaneously postmenopausal (PM, 40-50 years, $n=10)$. Subjects were admitted to the University Hospital General Clinical Research Center (GCRC) for a first-night sleep study conducted during a 24-hour, frequent blood sampling protocol.

Results: Despite similar estrogen concentrations in the YC (28 $\pm 4 \mathrm{pg} / \mathrm{ml})$ and OC $(34 \pm 6$ $\mathrm{pg} / \mathrm{ml})$ groups, OC women had reduced sleep efficiency $(79 \% \pm 2 \%)$ vs. YC $(87 \% \pm 3 \% ; p=$ 0.009). In the OVX and PM groups where estrogen concentrations were markedly different, sleep efficiency was also reduced vs. the YC group (OVX vs. YC, 79\% $\pm 3 \%$ vs. $87 \% \pm 3 \%, p=$ 0.05 ; PM vs. YC, $75 \% \pm 3 \%$ vs. $87 \% \pm 3 \%, p=0.007)$. Wake time was longer in the three older groups (103 \pm 10 minutes, $101 \pm 12$ minutes, $123 \pm 12$ minutes for OC, OVX, PM, respectively) vs. YC (63 \pm 13 minutes, $p<0.05)$. The number of stage shifts was positively associated with advancing age $(r h o=0.3, p<0.03)$ but not with estrogen concentration.

Conclusions: Aging-related sleep deficits in response to an experimental stressor occur in midlife women prior to menopause.

\section{INTRODUCTION}

$S_{\mathrm{n}}^{\mathrm{L}}$ LEeP COMPLAinTS ARE COMMON SYMPTOMS as the menopause approaches, presumably brought about by estrogen withdrawal and the accompanying hot flashes and night sweats. ${ }^{1-5}$ Although

the use of estrogen therapy (ET) to improve sleep in symptomatic women is widely accepted, clinical studies have demonstrated only relatively modest improvements, ${ }^{6,7}$ suggesting that underlying aging-related deficits may be involved in middle-age sleep impairment.
\end{abstract}

\footnotetext{
${ }^{1}$ School of Nursing, ${ }^{2}$ General Clinical Research Center, and ${ }^{3}$ Reproductive Sciences Program, University of Michigan, Ann Arbor, Michigan.

${ }^{4}$ School of Nursing, University of Texas at Austin, Austin, Texas.

This work was supported by NIH grants RO1-AG15083-04 and 5-MO1-RR-00042 and was conducted while J.L.L. was a Pfizer Postdoctoral Fellow.
} 
One of the biggest methodological drawbacks of sleep studies has been the use of middle-aged subjects inadequately defined for reproductive status and sex steroid exposure. ${ }^{8,9}$ Prior investigations of sleep in perimenopausal women where menstrual cycles are irregular have failed to characterize hormone levels or control for the potential soporific effects of progestogen as a function of oral contraceptive use and ovulatory status. $8,10,11$ In sleep studies of postmenopausal subjects, confounding factors presumed to mediate the estrogen milieu, such as years since menopause, ovariectomy, use of hormone therapy (HT), hot flash frequency, ovarian cysts, and body weight, have not been considered. 6,9,12,13 Confirmation by ultrasound of hysterectomy status with or without complete or partial oophorectomy has seldom been undertaken despite relatively high rates of inaccurate recall of surgical outcomes. ${ }^{14}$ Most importantly, the chronological age ranges in these postmenopausal study samples have varied by as much as 30 years.

To better distinguish aging effects per se from those due to the sex steroid environment, we examined sleep architecture in both young and middle-aged ovulatory subjects free of menopause complaints during a hospital sleep challenge. We used an experimental approach (frequent blood sampling) that has been shown previously to discriminate age and gender effects on laboratory sleep. ${ }^{15,16}$ We hypothesized that older cycling (OC), asymptomatic women with a similar estrogen profile would have greater sleep impairment in response to an episode of disturbed sleep than young cycling (YC) women. To characterize any menopause effect on aging impairments, we included two acyclic older groups, a spontaneously postmenopausal (PM) group and a group of ovariectomized women receiving ET (OVX) at doses comparable to midfollicular phase levels. We predicted that sleep function would be further compromised in the untreated PM group.

\section{MATERIALS AND METHODS}

\section{Subjects}

Before initiation of the study, approval of the clinical protocol was obtained from the University of Michigan Hospitals Institutional Review Board (IRB) for research with human subjects. All volunteers provided written informed consent. Fifty-one studies were conducted in the sleep laboratory of the General Clinical Research Center (GCRC) at the University of Michigan Hospitals in four groups of women differing by age or menopause status: 14 YC (20-30 years), 15 OC (40-50 years), 12 OVX (40-50 years), and 10 PM (40-50 years) women. Healthy volunteers were recruited by advertisement in local papers, bulletin board fliers, and from a website. All women met the following criteria: normal endocrine screen and no current history of smoking, medical or psychiatric illness, medication usage including oral contraceptives, benzodiazepines, antidepressants, psychostimulants or over-thecounter drugs or herbs, pregnancy or breast feeding in the past 6 months, sleep disorders, shift work, dieting, or excessive exercise or alcohol consumption. Women who reported symptoms of hot flashes and nights sweats were excluded. As sleep-disordered breathing has been reported in women with an elevated body mass index $(\mathrm{BMI}){ }^{17}$ we chose to exclude applicants with a BMI $\geq 32 \mathrm{~kg} / \mathrm{m}^{2}$. For the OC group, only those with regular menstrual cycles every 25-35 days with cycle-to-cycle variation of $\leq 3$ days were included. For the PM group, subjects had to report a spontaneous cessation of menses at least 12 months prior to study and no use of HT within 6 months of enrollment. For the OVX group, subjects were switched from oral ET to an estradiol $\left(E_{2}\right)$ patch $(0.1 \mathrm{mg}$ every 3 days) for at least 6 weeks prior to study. In all subjects, the results of screening values for hemoglobin, hematocrit, liver function, glucose, prolactin, follicle-stimulating hormone (FSH), and $\mathrm{E}_{2}$ were in expected ranges. Sample size was based on a power analysis using expected differences in estrogen concentrations in the three older groups, where an $n$ of 12 would provide $80 \%$ power.

\section{Sleep challenge}

Naive volunteers were admitted at 7 Am to the inpatient division of the GCRC of the University of Michigan Hospitals for a 24-hour intensive blood sampling protocol and sleep study conducted on the first night of admission. Blood was collected every 10 minutes via indwelling catheter between 9 AM on the day of admission to 9 AM the next day as part of a larger study of dynamic gonadotropin secretion during menopause. ${ }^{18} \mathrm{~A}$ less frequent blood sampling schedule 
has been shown to be an effective experimental paradigm to induce disturbed sleep in women over age $55 .{ }^{16}$

Subjects were ambulatory at will during the day and early evening in a private room with standard hospital lighting, then in bed with lights turned out between $11 \mathrm{PM}$ and $7 \mathrm{AM}$ of the following day. Caffeinated beverages were restricted after 1 PM. For each 10-minute sample, 2 $\mathrm{ml}$ of blood was drawn at the bedside from an indwelling, intravenous (i.v.) cannula inserted in a forearm vein, then flushed with heparinized saline. Subjects were allowed to sleep on their back or on the side opposite to the blood drawing arm, changing position at will. Volunteers were questioned in the morning about the quality of their sleep compared with normal $(1=$ same as normal, $2=\mathrm{OK}$ but less than normal, $3=$ poor) and whether they had experienced hot flashes or night sweats.

\section{Sleep monitoring}

After electrodes were applied at 9 PM, polysomnographic recordings were performed from 11 PM (lights out) to $7 \mathrm{AM}$ (lights on), for a total of 480 minutes, and scored using 30-second epochs according to the standard method established by Rechtschaffen and Kales. ${ }^{19}$ The recording montage was the following: left and right electro-oculogram, chin electromyogram, and electrode placement of C3/A2, C4/A1, O1/A2, and $\mathrm{O} 2 / \mathrm{A} 1$. The number of stage shifts (a sleep stage change from one epoch to the next) was reported for each recording. Sleep arousals (as defined in EEG Arousals: Scoring Rules and Examples) ${ }^{20}$ were scored, and the arousal index (number of arousals per hour of sleep) was reported for each study. Sleep latency was defined as the number of minutes from the time between lights off and the first epoch of any sleep stage. Total sleep was determined from the sum of sleep stages 1, 2, 3, 4, and REM sleep. Sleep efficiency was the calculated value of the total sleep time (of stages 1, 2, 3, 4, and REM) divided by the total sleep recording period (11 $\mathrm{PM}-7 \mathrm{AM})$, multiplied by 100. REM latency was defined as the time span between sleep onset and the occurrence of the first epoch of REM sleep. Sleep architecture indices obtained included percentage of time spent asleep in the different stages: awake, stage 1, stage 2, REM, and delta sleep (stages 3 and 4).

\section{Menstrual cycle parameters}

Cycling women were studied on a single day during the early follicular phase (cycle day $4 \pm 1$; day 1 = the first day of menses) of an ovulatory menstrual cycle. Three single blood draws were collected at weekly intervals after discharge for measurement of progesterone $(P)$ to determine ovulatory status in the menstruating volunteers ( $\mathrm{P} \geq 3 \mathrm{ng} / \mathrm{ml}$ on at least one occasion). Menstrual cycle length was determined from day 1 of menses to the day prior to the onset of the next menstrual period. Vaginal ultrasonography was performed to confirm the relevant ovarian function (e.g., ovarian volume, follicle size, number) (data not shown) or postsurgical status in each of the study groups.

\section{Reproductive hormone assays}

As a marker of reproductive aging, mean values of FSH were determined from the every-10minute blood draws, sampled over 24 hours. Mean values for $E_{2}$ were determined from four samples obtained at $9 \mathrm{AM}, 3 \mathrm{PM}, 1 \mathrm{AM}$, and $5 \mathrm{AM}$. Plasma $\mathrm{E}_{2}$ (assay sensitivity $5 \mathrm{pg} / \mathrm{ml}$ ) was measured by radioimmunoassay (RIA) (Diagnostic Products Corp., Los Angeles, CA). Intraassay and interassay coefficients of variation $(\mathrm{CV})$ for $\mathrm{E}_{2}$ were $7.8 \%$ and $16.2 \%$, respectively. For analysis, values below assay sensitivity were assigned the value of assay sensitivity. Plasma FSH was determined either by Delfia $^{\circledR}$ fluorometric immunoassay (IFMA) (Wallac Inc., Gaithersburg, MD) or Immulite ${ }^{\circledR}$ chemiluminescent assay (Diagnostic Products Corp.). The correlation of results measured by Delfia and Immulite was $r=$ $0.965(p<0.001)$. For both FSH assays, the limit of detection was $0.05 \mathrm{IU} / \mathrm{L}$.

\section{Statistical analysis}

A total of 63 subjects underwent the experimental protocol. Prior to analysis, data from 12 subjects were excluded for the following reasons: technical problems with equipment and noise control $(n=5)$, failure to ovulate during the study cycle $(n=1)$, inaccurate timing of the early follicular phase $(n=4)$, inappropriate ET dose $(n=1)$, and febrile episode during the protocol $(n=1)$, leaving 51 studies for analysis. Descriptive data are presented as mean \pm SE. The distribution of physiological variables was explored for normality by calculating skew and kurtosis 
before applying standard tests. As sleep and physiological data were skewed, nonparametric tests were performed. For the entire sample, correlational analyses between sleep parameters and age or $E_{2}$ was performed using Spearman's rho. Physiological and sleep parameters (continuous variables) in each group were compared using the Mann-Whitney test or the Kruskal Wallis ANOVA with post hoc Mann-Whitney test. Categorical variables between groups were compared with Fischer's exact test. $p$ values were derived using the two-tailed test conducted at the 0.05 level.

\section{RESULTS}

\section{Clinical characteristics}

There were no significant differences in body size characteristics across groups. Table 1 presents the clinical features of the four study groups. In addition to the expected age difference between the young and older groups, the PM group was 4 years older on average than the OC and OVX women $(p \leq 0.03)$. Both cycling groups demonstrated hormone characteristics consistent with ovulation during the study cycle. The OC group exhibited an endocrine profile that was typical of the early follicular phase of older ovulatory women. ${ }^{21} \mathrm{E}_{2}$ was similar, but mean $\mathrm{FSH}$ $(p=0.001)$ was higher, and menstrual cycle length was shorter $(p<0.02)$ vs. the YC group. There were no reports of hot flashes or night sweats among the older study participants. There were no differences between the four groups for perceived quality of sleep (chi-square $=5.0$, $p<0.2)$.

\section{Sleep architecture}

For the sample as a whole, average sleep efficiency during the experimental challenge was $80 \% \pm 1 \%$. Slow wave sleep was $46 \pm 3$ minutes, and REM latency was $104 \pm 8$ minutes. Advancing age was correlated with reduced total sleep minutes (rho $=-0.4, p=0.002)$, longer wake time ( $r h o=0.4, p=0.001)$, reduced sleep efficiency ( $r h o=-0.4, p=0.001$ ), and greater number of stage shifts $(r h o=0.3, p<0.03)$. In all women who had measurable $\mathrm{E}_{2}$ concentrations $>5 \mathrm{pg} / \mathrm{ml}(n=44)$, no association between $\mathrm{E}_{2}$ and any sleep parameter was found (Table 2). Additionally, we examined the correlation of $E_{2}$ with sleep parameters within each of the four groups separately. There was no association between $\mathrm{E}_{2}$ and any sleep parameter in any group $(p=N S)$.

Table 3 presents the sleep characteristics of the four study groups. Although latency to sleep onset was not different across groups, total sleep time, wake time, and sleep efficiency differed by age, with all three older groups demonstrating similar differences when compared with the YC group. In the YC group, 8 of 14 subjects (57\%) exhibited a sleep efficiency $>90 \%$ compared with 4 of 37 subjects $(11 \%)$ who were over age 40 (chi-square $=12.1, p=0.001$ ). Among the three groups of women over age 40, no differences were observed for any measure of REM or nonREM sleep.

\section{DISCUSSION}

We were able to demonstrate that middle-aged women, regardless of ovarian function, experienced greater sleep disturbance than younger

Table 1. Clinical Characteristics of Women by Menstrual Status

\begin{tabular}{|c|c|c|c|c|}
\hline & $\begin{array}{c}\text { Young cycling } \\
(Y C) \\
(\mathrm{n}=14)\end{array}$ & $\begin{array}{l}\text { Old cycling } \\
\qquad(\mathrm{OC}) \\
(\mathrm{n}=15)\end{array}$ & $\begin{array}{c}\text { Ovariectomy }+E T \\
(O V X+E T) \\
(\mathrm{n}=12)\end{array}$ & $\begin{array}{c}\text { Postmenopausal } \\
\text { (PM) } \\
(\mathrm{n}=10)\end{array}$ \\
\hline Age (years) & $24.1 \pm 1.0^{\mathrm{a}}$ & $45.5 \pm 0.9^{*}$ & $45.8 \pm 1.2^{*}$ & $49.4 \pm 0.6^{*, * *, * * *}$ \\
\hline $\mathrm{BMI}\left(\mathrm{kg} / \mathrm{m}^{2}\right)^{\mathrm{b}}$ & $23.9 \pm 0.9$ & $24.7 \pm 0.7$ & $24.4 \pm 0.7$ & $25.7 \pm 1.4$ \\
\hline Estradiol day $5(\mathrm{pg} / \mathrm{ml})$ & $28.1 \pm 3.7$ & $36.6 \pm 5.5$ & $80.0 \pm 13.4^{*, * *}$ & $6.8 \pm 1.7^{*, * *, * * *}$ \\
\hline FSH mean 24 hr (IU/L) & $4.3 \pm 0.3$ & $9.0 \pm 1.3^{*}$ & $37.1 \pm 5.1^{*, * *}$ & $79.9 \pm 11.0^{*, * *, * * *}$ \\
\hline Study cycle length (days) & $28.8 \pm 1.1$ & $25.6 \pm 0.6^{*}(n=13)$ & NA & NA \\
\hline Study cycle day & $5.1 \pm 0.3$ & $4.4 \pm 0.3(n=14)$ & NA & NA \\
\hline
\end{tabular}

${ }^{a}$ Values are mean \pm SE, determined on cycle day 5. Post hoc Mann-Whitney U analyses performed if ANOVA revealed significant group effect.

${ }^{b}$ BMI, body mass index; FSH, follicle-stimulating hormone; NA, not applicable.

${ }^{*} p \leq 0.02$ vs. YC; ${ }^{* *} p \leq 0.006$ vs. OC; ${ }^{* * *} p \leq 0.03$ vs. OVX+ET. 
Table 2. Spearman rho Correlations for Age and $E_{2}$ with Sleep Parameters

\begin{tabular}{lcr}
\hline & $\begin{array}{c}\text { Age } \\
(\mathrm{n}=51)\end{array}$ & $\begin{array}{c}E_{2}^{\mathrm{a}} \\
(\mathrm{n}=44)\end{array}$ \\
\hline Total sleep time (min) & $-0.4^{* *}$ & -0.2 \\
Wake time (min) & $0.4^{* * *}$ & 0.2 \\
\% Sleep efficiency & $-0.4^{* * *}$ & -0.2 \\
Total number of stage shifts & $0.3^{*}(n=47)$ & $0.05(n=41)$ \\
Total number of arousals & $0.1 \quad(n=45)$ & 0.1 \\
Arousals per hour & $0.2(n=45)$ & $(n=39)$ \\
Latency to sleep & 0.07 & 0.2 \\
REM latency & 0.2 & -0.03 \\
Time of stage 1 (min) & -0.1 & 0.2 \\
Time of stage 2 (min) & -0.2 & -0.04 \\
Time of stages 3 and 4 (min) & -0.06 & 0.06 \\
Time of REM (min) & -0.2 & -0.1 \\
\% Stage 1 & 0.006 & -0.2 \\
\% Stage 2 & 0.06 & 0.004 \\
\% Stages 3 and 4 & 0.5 & 0.2 \\
\% Sleep stage REM & -0.1 & -0.09 \\
\hline
\end{tabular}

${ }^{a}$ Includes women with measurable $\mathrm{E}_{2}$ concentrations $>5 \mathrm{pg} / \mathrm{ml}$. Seven PM women had $\mathrm{E}_{2}$ concentrations below the assay detection limit and were dropped from the analysis.

${ }^{*} p<0.05 ;{ }^{* *} p<0.01 ;{ }^{* *} p=0.001$.

controls during a first-night hospital sleep challenge. We observed a significantly longer wake time (at least 30 minutes longer on average) in all three older age groups vs. YC $(p<0.05)$. Deficits in sleep efficiency and deep sleep (stages 3-4) for the middle-aged women were similar to those re- ported for a group of volunteers more than two decades older exposed to a similar experimental protocol. ${ }^{16}$ Although data are lacking for women, studies of first-night effects on sleep architecture in young and middle-aged men reveal a similar degree of change in sleep parameters as those de-

Table 3. Effects of Age and Estrogen Status on Sleep Architecture

\begin{tabular}{|c|c|c|c|c|}
\hline & $\begin{array}{c}\text { Young cycling } \\
(Y C) \\
(\mathrm{n}=14)\end{array}$ & $\begin{array}{l}\text { Old cycling } \\
\qquad(\mathrm{OC}) \\
(\mathrm{n}=15)\end{array}$ & $\begin{array}{c}\text { Ovariectomy }+E T \\
(O V X+E T) \\
(\mathrm{n}=12)\end{array}$ & $\begin{array}{c}\text { Postmenopausal } \\
\text { (PM) } \\
(\mathrm{n}=10)\end{array}$ \\
\hline Latency to sleep (min) & $14.9 \pm 3.1^{\mathrm{a}}$ & $18.6 \pm 3.6$ & $16.2 \pm 5.5$ & $18.0 \pm 3.9$ \\
\hline Total sleep (min) & $416.9 \pm 13.0$ & $377.8 \pm 9.7^{* *}$ & $376.4 \pm 12.7^{*}$ & $354.9 \pm 14.9^{* *}$ \\
\hline Wake time (min) & $63.4 \pm 13.0$ & $102.8 \pm 9.7^{* *}$ & $101.4 \pm 12.4^{*}$ & $122.8 \pm 11.7^{* *}$ \\
\hline Sleep efficiency (\%) & $86.8 \pm 2.7$ & $78.7 \pm 2.0^{* *}$ & $78.8 \pm 2.6^{*}$ & $74.5 \pm 2.8^{* *}$ \\
\hline Number of stage shifts & $142.4 \pm 9.8$ & $146.5 \pm 6.5(n=13)$ & $164.5 \pm 14.8(n=11)$ & $177.4 \pm 12.0(n=9)$ \\
\hline Number of arousals & $69.7 \pm 8.1$ & $82.2 \pm 9.4(n=13)$ & $72.9 \pm 8.2(n=10)$ & $86.9 \pm 11.9(n=8)$ \\
\hline Arousals per hour & $10.0 \pm 1.2$ & $13.1 \pm 1.6(n=13)$ & $11.9 \pm 1.5(n=10)$ & $12.7 \pm 1.5(n=8)$ \\
\hline \multicolumn{5}{|l|}{ REM sleep ${ }^{b}$} \\
\hline REM latency & $82.9 \pm 10.5$ & $104.8 \pm 11.3$ & $125.0 \pm 25.1$ & $108.7 \pm 15.1$ \\
\hline Time of REM (min) & $91.3 \pm 9.3$ & $73.6 \pm 7.9$ & $66.5 \pm 9.9$ & $67.8 \pm 11.4$ \\
\hline \% Sleep stage REM & $21.7 \pm 2.0$ & $19.3 \pm 2.0$ & $17.4 \pm 2.3$ & $18.5 \pm 2.6$ \\
\hline \multicolumn{5}{|l|}{ Non-REM sleep } \\
\hline Time of stage 1 (min) & $55.1 \pm 8.9$ & $45.1 \pm 4.0$ & $46.1 \pm 4.7$ & $42.6 \pm 6.1$ \\
\hline Time of stage 2 (min) & $215.9 \pm 14.0$ & $224.3 \pm 10.9$ & $215.2 \pm 11.9$ & $197.6 \pm 8.7$ \\
\hline Time of stages 3 and 4 (min) & $54.5 \pm 6.3$ & $34.7 \pm 4.9$ & $48.7 \pm 6.8$ & $47.0 \pm 5.7$ \\
\hline$\%$ Stage 1 & $13.6 \pm 2.2$ & $12.1 \pm 1.2$ & $12.5 \pm 1.5$ & $12.4 \pm 2.0$ \\
\hline$\%$ Stage 2 & $51.4 \pm 2.6$ & $59.3 \pm 2.3$ & $57.3 \pm 3.1$ & $56.1 \pm 2.4$ \\
\hline$\%$ Stages 3 and 4 & $13.0 \pm 1.5$ & $9.2 \pm 1.4$ & $12.7 \pm 1.6$ & $13.0 \pm 1.3$ \\
\hline
\end{tabular}

${ }^{a}$ Values are mean \pm SE. Post hoc Mann-Whitney $U$ analyses performed if ANOVA revealed a significant group effect.

${ }^{b} R E M$, rapid eye movement sleep.

${ }^{*} p \leq 0.05$ vs. YC; ${ }^{* *} p \leq 0.01$ vs. YC. 
scribed here for our OC and YC groups. ${ }^{15}$ In other studies of acclimated subjects, middle-aged men and women demonstrate more difficulties than younger participants in adapting to sleep-wake cycle challenges. ${ }^{22}$ Taken together, these data suggest that middle-aged individuals are less able to accommodate to a novel sleep challenge than their younger counterparts and demonstrate sleep deficits at an earlier age than previously believed.

Our findings fail to support the popular view that estrogen plays an important role in sleep enhancement. Sleep architecture in the older ovariectomized subjects receiving ET did not approximate that observed in the young women or differ from that of the postmenopause group where estrogen concentrations were near the limits of assay detection. As all older subjects were asymptomatic for hot flashes, two alternate explanations could account for the age-related sleep impairments in the older cycling, estrogen-replete women. Although individuals who reported sleep complaints, stressful life events, or use of psychoactive medications were excluded from study, it is not known to what extent higher background levels of psychosocial stress in the middle-aged group could have explained the sleep deficits, as no assessments of psychosocial well-being or quality of life were performed. If this were the case, however, one might have predicted enhanced sleep during the study (i.e., catch-up sleep) as a result of chronic sleep deprivation. Alternatively, it could be argued that the younger controls may have been sleep deprived owing to work or school activities, thus leading to greater sleep efficiency during the study. The use of a daily sleep log to track sleep habits in the week prior to the study would have more effectively addressed this question.

It is also possible that the older cycling women experienced night sweats or hot flashes that were not significant enough to have been recalled subjectively but intense enough to have disrupted the continuity of sleep. As reviewed by Shaver, ${ }^{23}$ the evidence from mostly cross-sectional studies of subjects during polysomnographic monitorings demonstrates that sleep disturbances occur mainly in menopausal women most bothered by hot flash/sweat activity. Without skin conductance recordings to confirm hot flash episodes, this cannot be ruled out.

Our conclusions are in contrast to those reached by Moe et al., ${ }^{24}$ who used a similar stress paradigm in elderly women (mean age 70 years) taking ET for more than 10 years. In that study, no group differences were observed in sleep quality, but the ET group experienced less of a decline from baseline. To what extent the advanced age and years since menopause may have contributed to this effect is not known. Notably, the sleep efficiencies of $78 \%$ and $74 \%$ in the elderly ET users and control women, respectively, during blood sampling were strikingly similar to what we found in the unconditioned middle-aged groups with varying ovarian function.

In placebo-controlled trials using objective measures of sleep architecture, findings have been equivocal with respect to the beneficial impact of ET. Data from early studies conducted primarily with heterogeneous groups of clinic patients of varying ages, psychological status, menopause symptoms, history of estrogen use, and washout intervals $s^{6,25,26}$ have demonstrated decreased latency to sleep onset, decreased wakefulness after sleep onset, increased total sleep time, and enhanced REM sleep after the initiation of ET. As reviewed by Manber and Armitage, ${ }^{27}$ these trials were limited by the use of patient volunteers recruited from clinics where rates of menopause symptoms, insomnia, and depression are typically heightened compared to that of community-based samples, making it difficult to rule out secondary sleep benefits due to symptom relief.

Results from a more recent study ${ }^{28}$ have failed to corroborate these earlier trials when a randomized, double-blind, crossover clinical trial was used with postmenopausal volunteers from the general population, having more variable hot flash symptomatology. The use of an estrogen patch or gel for 3 months significantly reduced hot flashes and sleep complaints but did not improve any objective measure of sleep architecture assessed on the first night. Instead, the investigators demonstrated a significant reduction in limb movements in ET users and concluded that the benefits of ET on sleep are due to reduced muscle activity. A recent large epidemiological study, although failing to measure hormones, showed that the self-reported occurrence of menopause was not a predictor of objective sleep architecture. $^{29}$

Although longitudinal studies are needed, the findings from this small cross-sectional study add to the growing body of evidence that sleep architecture is not altered substantially by meno- 
pause. These findings, though provocative, require confirmation in larger populations well defined for reproductive states. Moreover, the relevance to natural sleep conditions of an experimental paradigm designed to induce disturbed sleep needs to be further defined. Future studies of the influence of middle age on sleep should take into account additional endocrine alterations that precede the menopause-related suppression in estrogen, including the age-related decline in growth hormone, androgens, and melatonin. The use of more comprehensive methods for systematically monitoring hot flashes, limb movements, and sleep architecture is warranted to better assess the neuroendocrine $\mathrm{dy}$ namics associated with gender differences in sleep changes with age.

\section{ACKNOWLEDGMENTS}

We thank Ann Brown, R.N., N.P., and Rudi Ansbacher, M.D., for conducting the vaginal ultrasounds, Alice Rolfes-Curl, B.A., and Vasantha Padmanabhan, Ph.D., for reproductive hormone analysis, the nursing staff of the University of Michigan General Clinical Research Center, and Beth Malow, M.D., for her review of the preliminary data. We are especially grateful to the women who served as research participants, some of whom were recruited from the University of Michigan Women's Health Registry.

\section{REFERENCES}

1. Shaver J, Giblin E, Lentz M, Lee K. Sleep patterns and stability in perimenopausal women. Sleep 1988;11: 556.

2. Woodward S, Freedman RR. The thermoregulatory effects of menopausal hot flashes on sleep. Sleep 1994;17:497.

3. Hollander LE, Freeman EW, Sammel MD, Berlin JA, Grisso JA, Battistini M. Sleep quality, estradiol levels, and behavioral factors in late reproductive age women. Obstet Gynecol 2001;98:391.

4. Kravitz HM, Ganz PA, Bromberger J, Powell LH, Sutton-Tyrrell K, Meyer PM. Sleep difficulty in women at midlife: A community survey of sleep and the menopausal transition. Menopause 2003;10:19.

5. Moe KE. Reproductive hormones, aging, and sleep. Semin Reprod Endocrinol 1999;17:339.

6. Schiff I, Regestein Q, Tulchinsky D, Ryan KJ. Effects of estrogens on sleep and psychological state of hypogonadal women. IAMA 1979;242:2405.
7. Antonijevic IA, Stalla GK, Steiger A. Modulation of the sleep electroencephalogram by estrogen replacement in postmenopausal women. Am J Obstet Gynecol 2000;182:277.

8. Clark AJ, Flowers J, Boots L, Shettar S. Sleep disturbances in mid-life. J Adv Nurs 1995;22:562.

9. Edinger JD, Glenn DM, Bastian LA, et al. Sleep in the laboratory and sleep at home II: Comparison of middle-aged insomnia sufferers and normal sleepers. Sleep 2001;24:761.

10. Baker A, Simpson S, Dawson D. Sleep disruption and mood changes associated with menopause. J Psychosom Res 1997;43:359.

11. Owens JF, Matthews K. Sleep disturbance in healthy middle-aged women. Maturitas 1998;30:41.

12. Polo-Kantola P, Erkkola R, Helenius H, Irjala K, Polo $\mathrm{O}$. When does estrogen replacement therapy improve sleep quality? Am J Obstet Gynecol 1998;178:1002.

13. Peterson DD, Schmidt RM. Longitudinal and crosssectional analysis of healthwatch data with a subset of perimenopausal women and matched controls. J Gerontol Biol Sci 1999;54A:B160.

14. Horwitz RI, Yu EC. Problems and proposals for interview data in epidemiological research. Int J Epidemiol 1985;14:463.

15. Hirshkowitz M, Moore CA, Hamilton CR III, Rando $\mathrm{KC}$, Karacan I. Polysomnography of adults and elderly: Sleep architecture, respiration, and leg movement. J Clin Neurophysiol 1992;9:56.

16. Vitiello MV, Larsen LH, Moe KE, Borson S, Schwartz RS, Prinz PN. Objective sleep quality of healthy older men and women is differentially disrupted by nighttime periodic blood sampling via indwelling catheter. Sleep 1996;19:304.

17. Kripke DF, Ancoli-Isreal S, Klauber MR, Wingard DL, Mason WJ, Mullaney DJ. Prevalence of sleep-disordered breathing in ages 40-64 years: A populationbased survey. Sleep 1997;20:65.

18. Reame N. Gonadotropin changes in the perimenopause. In: Lobo RA, ed. Perimenopause. New York: Springer-Verlag, 2001:157.

19. Rechtschaffen A, Kales A. A manual of standardized terminology, techniques, and scoring system for sleep states of human subjects. Los Angeles: Brain Information Service/Brain Research Institute, UCLA, 1968.

20. EEG arousals: Scoring rules and examples: A preliminary report from the Sleep Disorders Atlas Task Force of the American Sleep Disorders Association. Sleep 1992;15:173.

21. Reame NE, Wyman TL, Phillips DJ, de Kretser DM, Padmanabhan V. Net increase in stimulatory input resulting from a decrease in inhibin $\mathrm{B}$ and an increase in activin A may contribute in part to the rise in follicular phase follicle-stimulating hormone of aging cycling women. I Clin Endocrinol Metab 1998;83:3302.

22. Carrier J, Land S, Buysse DJ, Kupfer DJ, Monk TH. The effects of age and gender on EEG power spectral density in the middle years of life (ages 20-60 years old). Psychophysiology 2001;38:232. 
23. Shaver JLF. Sleep problems. I. Insomnia and sleepdisordered breathing. Menopause Manag 2000;9:20.

24. Moe KE, Larsen LH, Vitiello MV, Prinz PN. Estrogen replacement therapy moderates the sleep disruption associated with nocturnal blood sampling. Sleep 2001;24:886.

25. Erlik Y, Tataryn IV, Meldrum DR, Lomax P, Bajorek JG, Judd HL. Association of waking episodes with menopausal hot flushes. IAMA 1981;245:1741.

26. Scharf MB, McDannold MD, Stover R, Zaretsky N, Berkowitz DV. Effects of estrogen replacement therapy on rates of cyclic alternating patterns and hotflush events during sleep in postmenopausal women: A pilot study. Clin Ther 1997;19:304.

27. Manber R, Armitage R. Sex, steroids, and sleep: A review. Sleep 1999;22:540.

28. Polo-Kantola P, Erkkola R, Irjala K, Pullinen S, Virtanen I, Polo O. Effect of short-term transdermal estro- gen replacement therapy on sleep: A randomized, double-blind crossover trial in postmenopausal women. Fertil Steril 1999;71:873.

29. Young, T, Rabago D, Zgierska, A, Austin D, Finn L. Objective and subjective sleep quality in premenopausal, perimenopausal, and postmenopausal women in the Wisconsin sleep cohort study. Sleep 2003; 26:667.

Address reprint requests to: Jane L. Lukacs, Ph.D., M.S.N. School of Nursing 400 North Ingalls Building, Suite 2238 University of Michigan Ann Arbor, MI 48109-0482

E-mail: lukacsj@umich.edu 
This article has been cited by:

1. Nancy Fugate Woods, Kathleen Smith-DiJulio , Donald B. Percival , Eunice Y. Tao , Heather J. Taylor, Ellen Sullivan Mitchell . 2007. Symptoms during the Menopausal Transition and Early Postmenopause and Their Relation to Endocrine Levels over Time: Observations from the Seattle Midlife Women's Health StudySymptoms during the Menopausal Transition and Early Postmenopause and Their Relation to Endocrine Levels over Time: Observations from the Seattle Midlife Women's Health Study. Journal of Women's Health 16:5, 667-677. [Abstract] [PDF] [PDF Plus] 